\title{
Experimental study on hydrodynamic behaviors of high-speed gas jets in still water
}

\author{
Zhenqing Dai • Boyi Wang · Longxi Qi • \\ Honghui Shi
}

Received: 9 August 2005 / Revised: 25 January 2006 / Accepted: 3 April 2006 / Published online: 5 September 2006 (C) Springer-Verlag 2006

\begin{abstract}
The present paper describes experimental investigation on the flow pattern and hydrodynamic effect of underwater gas jets from supersonic and sonic nozzles operated in correct- and imperfect expansion conditions. The flow visualizations show that jetting is the flow regime for the submerged gas injection at a high speed in the parameter range under consideration. The obtained results indicate that high-speed gas jets in still water induce large pressure pulsations upstream of the nozzle exit and the presence of shock-cell structure in the over- and under-expanded jets leads to an increase in the intensity of the jet-induced hydrodynamic pressure.
\end{abstract}

Keywords Underwater gas jet . Flow pattern . Pressure pulsation - Imperfect expansion nozzle . Shock-cell structure

\section{Introduction}

Investigation on the hydrodynamic behaviors of submerged gas jets and their effects concerns quite a wide range of natural and engineering processes such as volcano eruption in deep seas, ice prevention in lakes, water destratification in reservoirs, aeration wastewater treatment, underwater cutting, pneumatic steelmaking and so on [1-3]. In addition, noise generation by

Z. Dai $\cdot$ B. Wang $(\varangle) \cdot$ L. Qi $\cdot$ H. Shi

LNM, Institute of Mechanics, Chinese Academy of Sciences,

Beijing, 100080, China

e-mail: wby@imech.ac.cn

H. Shi

College of Mechanical Engineering and Automation, Zhejiang Sci-Tech University, Hangzhou, 310018, China underwater gas jets is also of interest for some applications [4]. A better understanding of relevant phenomena and mechanisms in the submerged gas exhaust systems is imperative for optimizing various industrial processes. As an example, so-called injection metallurgy emerged in 1980s due to its greater metallurgical and economic advantages. Since then gas stirring by the injection of an inert or reactive gas (or even carried with special particles) into melts is widely used in converters and ladles, where top-, bottom-, sidewall-blowing or their combination is employed for strong mixing and large reaction rates. During the development of studies regarding metallurgical operations, the severe wear of refractory near the tuyere tip through which a submerged gas jet is injected has become one of the main research topics $[5,6]$. Concerning the problem of refractory erosion, various water-model experiments were carried out and the phenomenon of pressure oscillations, described with the term of back-attack, was observed in the liquid as well as inside the tuyere $[7,8]$. However, in the previous water-model experiments the investigators used simple cylindrical or convergent conical nozzles from which the gas discharges at a sound speed. Under these operation conditions, the flow regime of submerged jets is bubbling or transition to jetting although nominal Mach numbers at the tuyere exit (NMa) may be greater than unity by increasing the gas stagnation pressure above its critical value. These authors concluded that, after the transition from the bubbling to jetting regime, the backattack pressure takes a minor change as the nominal Mach number increases up to $\mathrm{NMa} \approx 3$ [6-9]. The present work attempts to study the hydrodynamic behaviors of underwater high-speed gas jets issuing from horizontally mounted nozzles operating in correct- and imperfect- expansion conditions. In the recent years, Qi et al. 
[10] and Shi et al. [11] performed experimental measurements respectively for the average and fluctuating pressures inside underwater high-speed gas jets. In this work, we focus on the upstream pressure oscillations induced by supersonic and sonic gas jets in quiescent water, especially on those shock- associated phenomena and effects. This research will be benefit to the applications of underwater jet-propulsion vehicles.

In general, submerged gas injection results in a complex two-phase flow pattern which can be distinguished into two different regimes: bubbling and jetting. The jetting regime is dominant in the flow system when the gas discharges at a high speed (for instance, up to sound speed or higher). For a supersonic or sonic gas jet in still water, as shown schematically in Fig. 1, a gas core is surrounded by a gas-water mixing layer which consists of a drop layer and a bubble layer. When these high-speed gas jets discharge from an imperfectly expanded nozzle, a shock-cell system then appears in the flow field where the detailed structure depends on the Mach number and the pressure ratio at the nozzle exit. Ozawa and Mori once obtained the shock pattern by still photographs with illumination of microflash [12]. The hydrodynamic behaviors of the submerged gas jets and their effects on upstream body attract many researchers and series of experimental investigations are needed in order to understand relevant phenomena and mechanisms.

\section{Experimental arrangement}

Figure 2 presents the schematic diagram of the experimental arrangement for high-speed gas jets in still water. The experimental set-up mainly consists of an air compressor, a gas tank, a pressure-adjusting valve, a solenoid valve, a rectangular water tank and a test nozzle assemblage supported in the water tank. The compressor (CZ-20/30FZK type) can provide working gas (air) at a required pressure up to $3 \mathrm{MPa}$. The large volume of the gas tank $\left(0.8 \mathrm{~m}^{3}\right)$ ensures a stable gas pressure in the

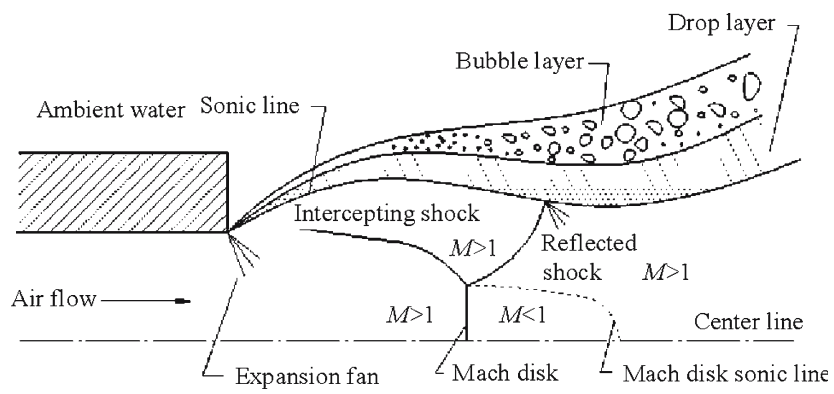

Fig. 1 Sketch of underwater high-speed gas jet at strongly imperfect-expansion condition

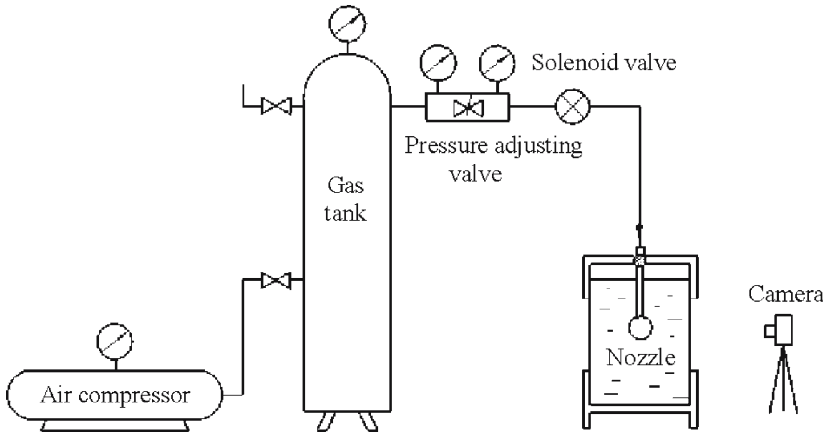

Fig. 2 Schematic diagram of experimental set-up for high-speed gas jets in still water

plenum chamber and a sufficient testing time of several seconds. The pressure-adjusting valve can regulate the gas stagnation pressure at the desired value. The open water tank is made of transparent plexiglass plates and has a size of $280 \mathrm{~cm}$ long, $50 \mathrm{~cm}$ wide and $55 \mathrm{~cm}$ high. Water with a level of $40 \mathrm{~cm}$ in the water tank is at atmospheric pressure and room temperature. The test nozzle is horizontally mounted at the submergence depth of $15 \mathrm{~cm}$. The key part of the nozzle assemblage with an outer diameter of $6.6 \mathrm{~cm}$ is the contoured nozzles. They are either convergent-divergent (Laval) nozzles for the supersonic case or monotonically convergent nozzles for the sonic case. All the nozzles take the same throat diameter of $0.43 \mathrm{~cm}$. For the test assemblage, there are four exchangeable nozzles having different area ratios of exit to throat. At the nozzle exit, the design Mach number (or Mach number based on correctly-expanded isentropic flow equations) Ma takes the value of 1.0, 1.5, 1.75 and 2.0, respectively. These four nozzles are elaborated, especially for their sizes of the throat and exit diameters. Moreover, before tests, each nozzle is carefully calibrated by using a total pressure probe in the design operation condition. As mentioned before, we considered three operation conditions for the supersonic nozzle (full-, over- and under-expansion) and two operation conditions for the sonic nozzle (full- and under-expansion). Usually, the imperfect-expansion index is defined by the ratio of the nozzle-exit pressure $\left(p_{\mathrm{e}}\right)$ to the ambient pressure $\left(p_{\mathrm{a}}\right)$. For each nozzle of the specified design exit Mach number Ma, the required operation condition can be implemented by adjusting the chamber pressure to be equal to, lower or higher than its critical value for the full-expansion operation.

In this work, free nozzle set-up was used owing to its advantage of recording the jet flow fields and measuring the pressure oscillations on the sidewall of the nozzle assemblage. Figure 3 shows the measurement arrangement for the flow visualization and pressure measurement. Clearly, by a baffle of the height $50 \mathrm{~cm}$, the water 


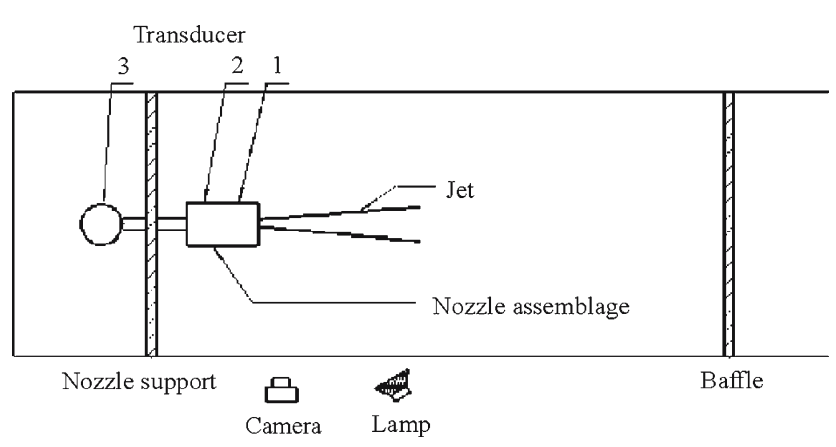

Fig. 3 Top view of water tank and measurement arrangement

tank was divided into two compartments where the large one was filled with tap water and the small one was empty for overflow. It is seen in Fig. 3 that the water pressures on the nozzle sidewall were measured by two electric-resistance pressure transducers 1 and 2 (NS-2 type) and the gas pressure in the nozzle plenum chamber was measured by an electric-capacity pressure transducer 3 (Setra 280E type). The data acquisition and analysis was done by using a high-speed dynamic test apparatus (DH5932 type). In addition, a Germany made digital camera (BASLER A602f type) was employed to observe the flow pattern of underwater gas jets. This digital camera was operated at a framing rate of $1 / 60 \mathrm{~s}$ during $5 \mathrm{~s}$ for one run. The taken images were processed by a personal computer.

\section{Measurement results}

The evolution process of high-speed air jets in still water was visualized by means of the digital camera. A typical image shows the instantaneous pattern of the flow

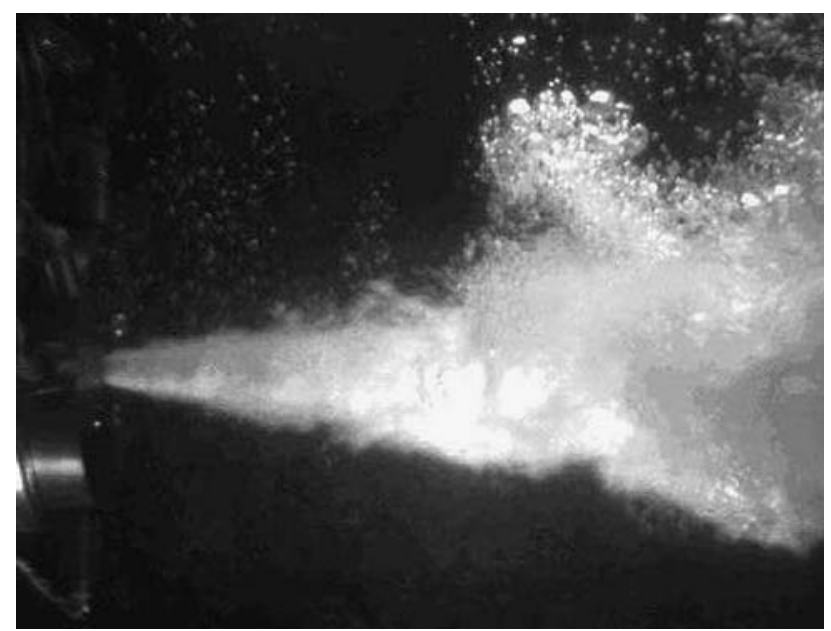

Fig. 4 Image of instantaneous flow pattern of high-speed air jet in still water field in Fig. 4. It is found that, due to great injection momentum, a continuous stream of gas issues from the submerged nozzle and the jet starts to break up into bubbles far downstream. Based on our measurements, the forward penetration of supersonic gas jets under consideration ranges from 50 to 100 diameters of the nozzle exit. The swarms of free bubbles formed in the jet front region rise up due to the action of buoyancy force. This causes breaking and sloshing of the free water surface. It is also found from Fig. 4 that some discrete bubbles appear in the proximity of the nozzle exit. In addition, the high-speed cinematography indicates that the gaswater interface is unstable, which may be attributed to the helical instability of supersonic gas jets in liquid [13].

Two typical pressure curves on the sidewall upstream of the nozzle exit, recorded by the transducer 1 , are plotted for the $\mathrm{Ma}=2.0$ case in Fig. 5. Figure 5a,b correspond to the cases with and without water in the water tank, respectively. Clearly, when this tank is filled by water, the submerged gas jet induces apparent hydrodynamic pressures upstream of the nozzle exit. It is shown in Fig. 5a that some large pulsations superpose small fluctuations where the latter ones can be considered as the jet noise. This is a distinctive feature of the underwater gas jets since there is only background noise in the empty tank case (see Fig. 5b). In order to determine which mechanism responds to the observed pressure oscillation phenomenon, we once covered the water surface with a flat plate and suppress the sloshing waves. Comparison between the measured pressures with and without the cover plate indicates that the free surface has little influence on the recorded pressure oscillations. These discrete pressure pulsations induced by the supersonic gas jets are similar to those so-called back-attack pressures in the previous experimental studies on injection metallurgy. The pressures in our tests were measured on the nozzle sidewall upstream of its exit but the pressures in their tests on the nozzle exit (cf. Ref. [14]).

As mentioned before, we focus specially on the upstream pressure oscillations associated with shock-cell structure in the underwater supersonic or sonic gas jets. Figure $6 a$, b show the pressure curves, recorded by transducer 1, for the nozzle with the design Mach number $\mathrm{Ma}=2.0$ operated in the over- and under-expansion condition where their corresponding pressure ratio is 0.8 and 1.2, respectively. Compared with the case of the nozzle with $\mathrm{Ma}=2.0$ operated in the full-expansion condition (see Fig. 5a), the frequency of pressure pulsation increases in the over-expansion case but decreases in the under-expansion case. However, compared to the fullexpansion case, the pulsation amplitude decreases in the over-expansion case but increases in the under-expansion case. 

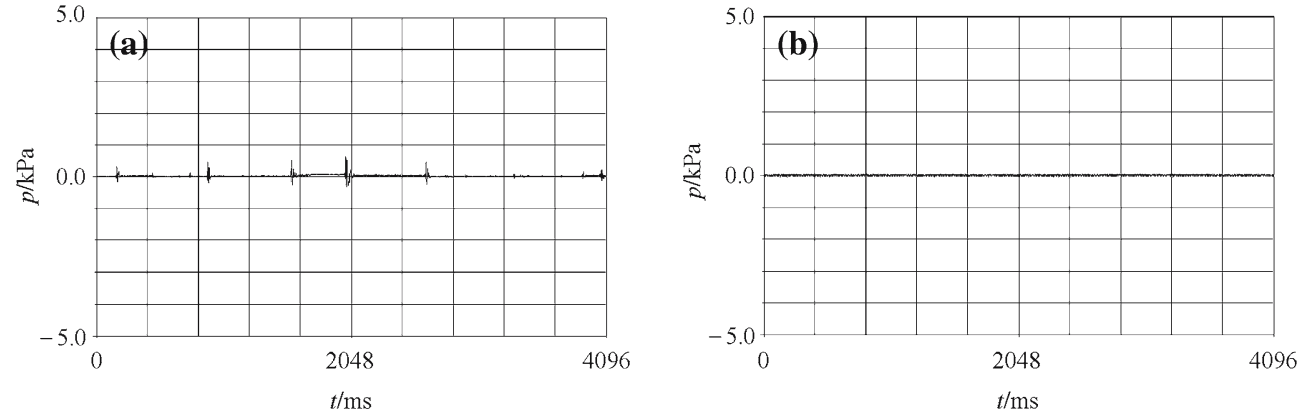

Fig. 5 Recorded oscillation curve of upstream sidewall pressure for Ma $=2.0$ nozzle at full-expansion operation. a Air jet in water; b air jet in air
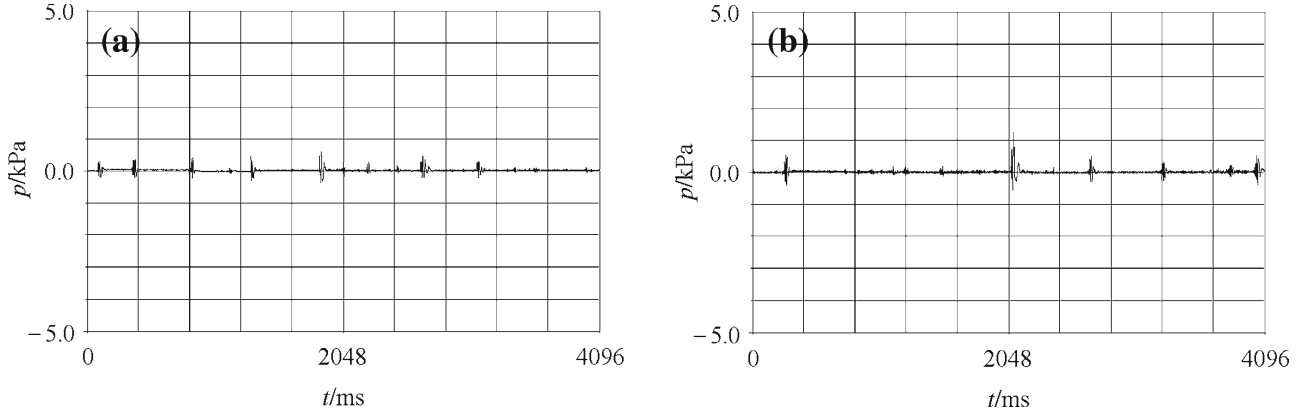

Fig. 6 Recorded oscillation curve of upstream sidewall pressure for Ma $=2.0$ nozzle. a Over-expansion operation; b under-expansion operation
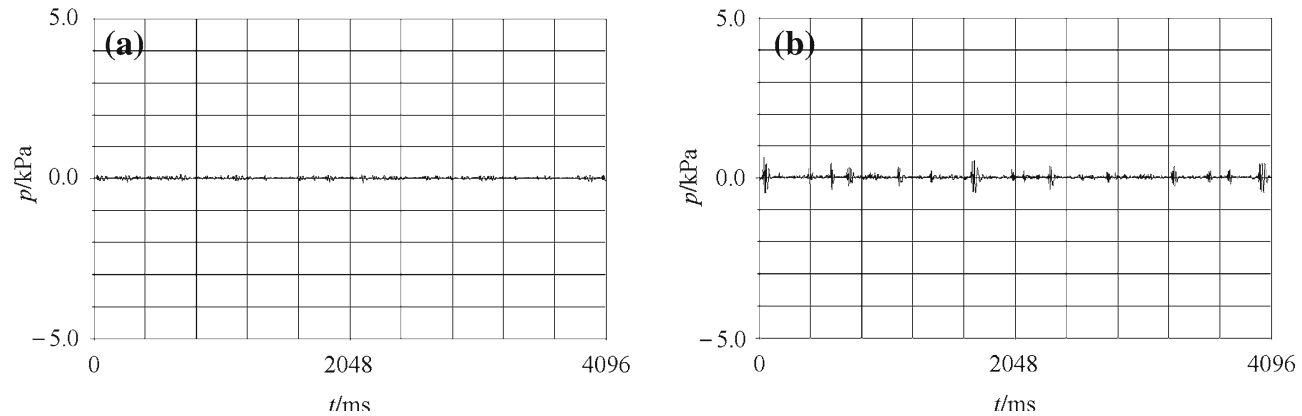

Fig. 7 Recorded oscillation curve of upstream sidewall pressure for Ma $=1.0$ nozzle. $\mathbf{a} p_{\mathrm{e}} / p_{\mathrm{a}}=1.0 ; \mathbf{b} p_{\mathrm{e}} / p_{\mathrm{a}}=5.0$
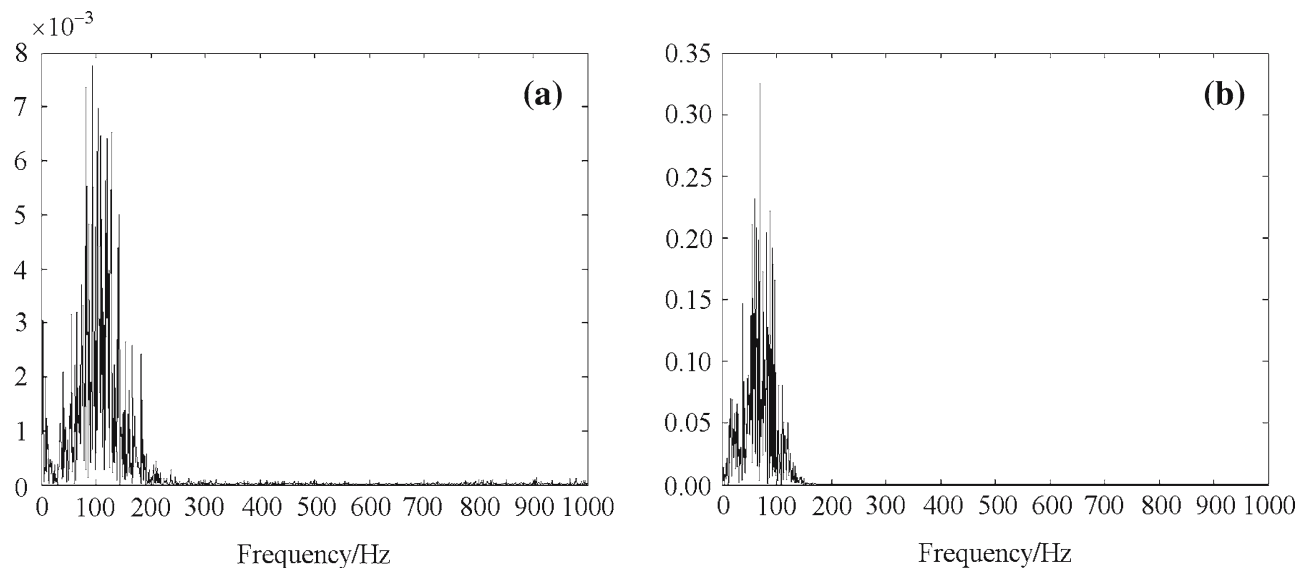

Fig. 8 Power spectrum of sidewall pressure induced by underwater imperfectly-expended gas jets. a $\mathrm{Ma}=2.0$ and $p_{\mathrm{e}} / p_{\mathrm{a}}=1.2$; b $\mathrm{Ma}=1.0$ and $p_{\mathrm{e}} / p_{\mathrm{a}}=5.0$ 
Figures 7a,b present the oscillation curves of upstream sidewall pressure, recorded by transducer 1 , respectively for the full- and under-expanded sonic nozzle. Contrary to the weak imperfect-expansion conditions for the supersonic case, strong under-expansion condition was considered for the sonic case. This operation condition $\left(p_{\mathrm{e}} / p_{\mathrm{a}}=5\right)$ corresponds to a nominal Mach number $\mathrm{NMa}=2.12$. Clearly, the behavior of pressure pulsation for the $\mathrm{NMa}=2.12$ sonic nozzle in the under-expansion condition is different from that for the $\mathrm{Ma}=2.0$ supersonic nozzle in the full-expansion condition.

In addition, it is noted in Figs. 5,6 and 7 that, due to the jet instability, the pressure pulsations are random events and some form of statistical treatment of the data is necessary. One simple measure of the intensity of the hydrodynamic effect is the r.m.s. value of the pressure oscillations. It is a measure of the mechanical power transferred from the gas jet to the nozzle wall. Besides, spectral analysis is also important for the study of stochastic behaviors of the submerged jets. By means of the fast Fourier transformation of the pressure data, some typical power spectra are presented in Fig. 8. It indicates that the spectral components of the mechanical power mainly distribute in the frequency band of $0-300 \mathrm{~Hz}$ and $0-200 \mathrm{~Hz}$ respectively for the supersonic and sonic case in the range of the operation parameters under consideration.

For the supersonic case, the r.m.s values of the pressure oscillations obtained by two transducers on the nozzle sidewall are given in Figs. 9a,b,c, respectively for the three tested nozzles with the design exit Mach number of $\mathrm{Ma}=1.5,1.75$ and 2.0. It is shown that, for the same Mach number, the induced hydrodynamic effect by the supersonic jets discharged from the imperfect expansion nozzles is always greater than that from the correct-expansion nozzles. Clearly, the shock-cell structure in the over- or under-expanded jets results in this enhancement in the intensity of the hydrodynamic pressures.

Finally, similar results are given in Fig. 10 for the sonic case. As pointed before, the exit pressure ratio in this case is greater than that in the supersonic case above. Here the nominal Mach number for $p_{\mathrm{e}} / p_{\mathrm{a}}=3$ case is $\mathrm{NMa}=1.79$. Obviously, the r.m.s. value of the pressure oscillations increases with increasing the pressure ratio at the nozzle exit.

\section{Summary}

Experimental investigation is performed on the behaviors of underwater gas jets issuing from supersonic and sonic nozzles operated in the full-, over- and under-
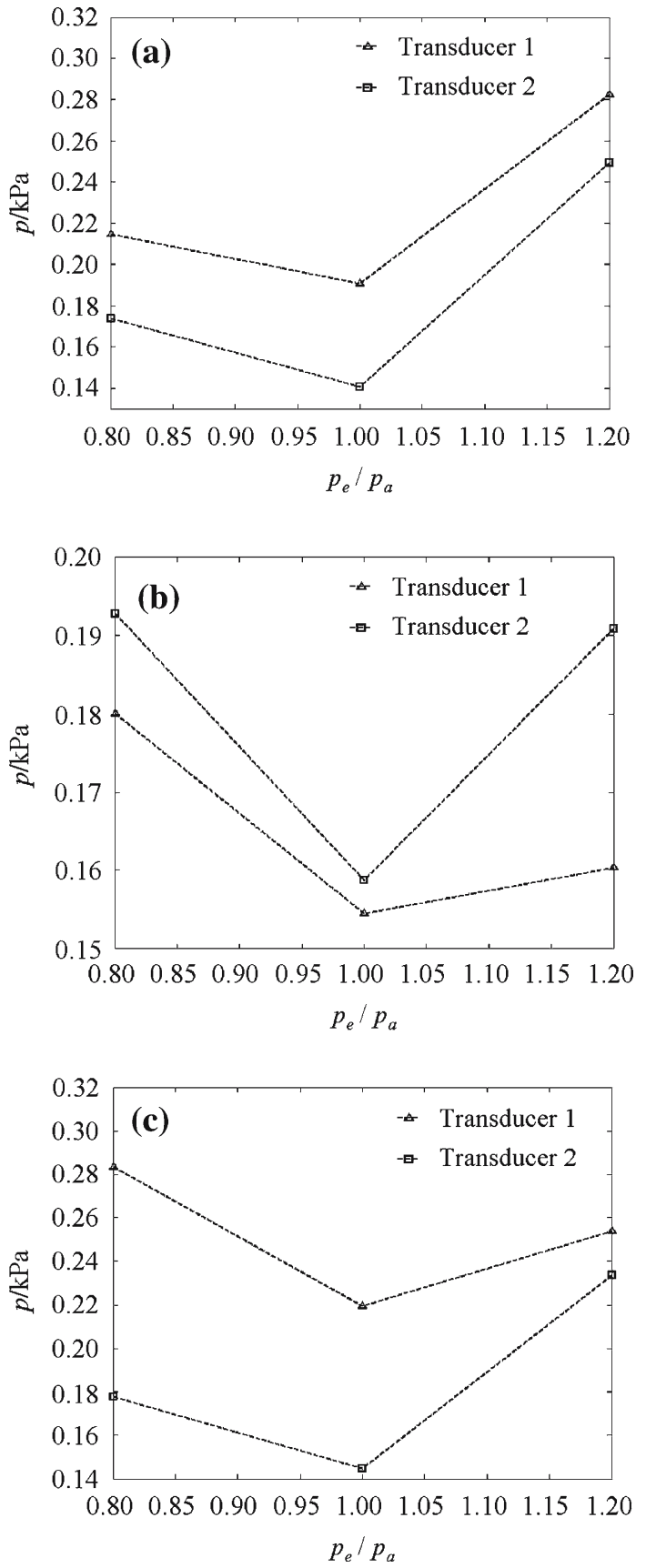

Fig. 9 The r.m.s. value of sidewall pressure oscillations induced by underwater supersonic gas jets. a $\mathrm{Ma}=1.5 ; \mathbf{b} \mathrm{Ma}=1.75 ; \mathbf{c} \mathrm{Ma}=2.0$

expansion conditions. The flow visualization shows that the jetting is the flow regime for the high-speed gas jets operated in the parameter range under consideration. The pressure measurements reveal a distinctive feature of the submerged high-speed gas jets: large discrete pulsations superpose small continuous fluctuations for the upstream sidewall pressures. In addition, the obtained results indicate that the intensity of the hydrodynamic pressure increases due to the presence of the 


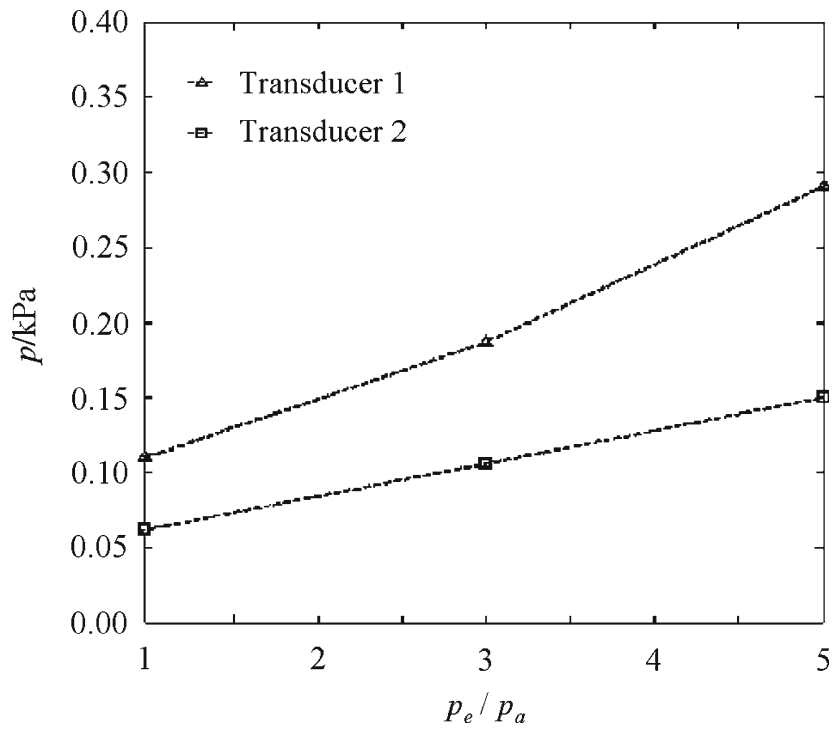

Fig. 10 The r.m.s. value of sidewall pressure oscillations induced by underwater sonic gas jets

shock-cell structure in the over- or under-expanded jets. Of course, further study is needed on the quantitative analysis of the shock-associate phenomena in the underwater supersonic gas jets.

\section{References}

1. Rensen J., Roig V.: Experimental study of the unsteady structure of a confined bubble plume. Int. J. Multiph. Flows 27, 1431 (2001)

2. Matsumoto, O., Sugihara, M., Miya, K.: Underwater cutting of reactor core internals by $\mathrm{CO}$ laser using local-dry-zone creating nozzle. J. Nucl. Sci. Technol. 29, 1074 (1992)
3. Bisio, G., Rubatto, G.: Process improvements in iron and steel industry by analysis of heat and mass transfer. Energy Convers. Manage. 43, 205 (2002)

4. Gavigan, J.J., Watson, E.E., King, W.F.III: Noise generation by gas jets in a turbulent wake. J. Acoust. Soc. Am. 56, 1094 (1974)

5. Fabritius, T.M.J., Mure, P.T., Harkki, J.J.: The determination of the minimum and operational gas flow rates for sidewall blowing in the AOD-Converter. ISIJ Int. 43, 1177 (2003)

6. Yang, Q.X., Gustavsson, H.: Effects of gas jet instability on refractory wear; a study by high-speed photography. Scand. J. Metall. 21, 15 (1992)

7. Aoki, T., Masuda, S., Hatono, A., Taga, M.: Characteristics of submerged gas jets and a new type bottom blowing tuyere. In: Wraith A.E. (ed) Injection Phenomena in Extraction and Refining, New Castle, April 21-22, pp.A1-A36 (1982)

8. Taylor, I.F., Wright, J.K., Philp, D.K.: Transient pressure and vibration events resulting from high speed gas injection into liquids. Can. Inst. Min. Metall. 27, 293 (1988)

9. Yang, Q.X., Gustavsson, H., Burstrom, E.: Erosion of refractory during gas injection - a cavitation based model. Scand. J. Metall. 19, 127 (1990)

10. Qi, L.X., Chao, Y., Wang, B.Y.: Experimental study of underexpanded sonic air jets in water. Acta Mech. Sin. 32, 667 (2000) (in Chinese)

11. Shi, H.H., Wang, B.Y., Qi, L.X., Li, Q., Yang, B.: The measurement of fluctuating pressures induced by submerged high-speed gas jet. In: Proceedings of The 4th International Symposium on Advanced Fluid Information and Transdisciplinary Fluid Integration, Sendai, Japan, November 11-12, pp. 77-81 (2004)

12. Ozawa, Y., Mori, K.: Characterization of jetting observed in gas injection into liquid. Trans. ISIJ 23, 765 (1983)

13. Chen, K., Richter, H.J.: Instability analysis of the transition from bubbling to jetting in a gas injected into a liquid. Int. J. Multiph. Flows 23, 699 (1997)

14. Cho, Y.W., Yoon, J.K.: Physical shielding by shrouding gas jet issuing horizontally into a liquid. SCANINJECT IV, Lulea, Sweden, June 11-13, pp. 4:1-4:19 (1986) 